\title{
Medical and Technical Protocol for Automatic Navigation of a Wireless Device in the Carotid Artery of a Living Swine Using a Standard Clinical MRI System
}

\author{
Sylvain Martel ${ }^{1}$, Jean-Baptiste Mathieu ${ }^{1}$, Ouajdi Felfoul ${ }^{1}$, Arnaud Chanu ${ }^{1}$, \\ Eric Aboussouan ${ }^{1}$, Samer Tamaz ${ }^{1}$, Pierre Pouponneau ${ }^{1}$, L'Hocine Yahia ${ }^{2}$, \\ Gilles Beaudoin ${ }^{3}$, Gilles Soulez ${ }^{3}$, and Martin Mankiewicz ${ }^{1}$
}

\author{
${ }^{1}$ NanoRobotics Laboratory, Department of Computer Engineering and Institute of \\ Biomedical Engineering, École Polytechnique de Montréal (EPM), Campus de l'université de \\ Montréal, P.O. Box 6079, Station Centre-ville, Montréal (Québec), Canada H3C 3A7 \\ ${ }^{2}$ Laboratoire d'Innovation et d'Analyse de la Bioperformance (LIAB), École Polytechnique \\ de Montréal, Montréal, (Québec), Canada \\ ${ }^{3}$ CHUM-Hôpital Notre-Dame Département de radiologie, Pavillon Lachapelle (CHUM) \\ Bureau C-1077 1560 Sherbrooke est Montréal (Québec) Canada H2L 4M1 \\ \{Sylvain.Martel, Jean-Baptiste.Mathieu, Ouajdi.Felfoul, \\ Arnaud. Chanu, Eric. Aboussouan, Samer. Tamaz, Pierre.Pouponneau, \\ L'Hocine Yahia, Martin.Mankiewicz\} @polymtl.ca, \\ \{Gilles.Beaudoin, Gilles.Soulez\} @umontreal.ca
}

\begin{abstract}
Astract. A $1.5 \mathrm{~mm}$ magnetic sphere was navigated automatically inside the carotid artery of a living swine. The propulsion force, tracking and real-time capabilities of a Magnetic Resonance Imaging (MRI) system were integrated into a closed loop control platform. The sphere was released using an endovascular catheter approach. Specially developed software is responsible for the tracking, propulsion, event timing and closed loop position control in order to follow a 10 roundtrips preplanned trajectory on a distance of $5 \mathrm{~cm}$ inside the right carotid artery of the animal. Experimental protocol linking the technical aspects of this in vivo assay is presented. In the context of this demonstration, many challenges which provide insights about concrete issues of future nanomedical interventions and interventional platforms have been identified and addressed.
\end{abstract}

Keywords: Magnetic resonance imaging, wireless, tracking, control, in vivo assay.

\section{Introduction}

The possibility of controlling magnetic particles using external magnetic fields opens the way for many medical applications. This potential has been acknowledged for many years now as, back in 1965, aneurism embolization studies were based on micron sized iron powders spatially confined with magnetic tipped catheters [1]. Ever since, magnetic drug delivery carriers have evolved and are now composed of state of the art nanoparticles such as stealth magnetoliposomes or smart polymer based 
magnetic particles [2-5]. Targeting of these particles is still performed using external magnets or magnetized needles or catheters. Magnetic targeting would greatly benefit from an increase of the role of computerized platforms to achieve automated control over the spatial distribution of the particles.

Stereotaxis for example proposes a computerized steering platform for catheters. Their magnetic tipped catheters can be steered using actuated external magnets [6]. Orientation of the catheters is controlled by computerized platform acting on the tilt angle of the external magnets. Proper corrective action is computed based on tracking information from X-ray projection images.

Computer controlled magnetic guidance platforms able to navigate magnetic particles to target the deepest recesses of the human body are currently under development.

In $[7,8]$, magnetic microdevices are navigated in vitro using a custom built coil apparatus relying on Helmholtz and Maxwell pairs. Optical tracking methods provide simple and effective solutions for the needs of theses applications. As a matter of fact, these microdevices are developed for future surgical procedures in transparent media such as the vitreous humor.

Optical tracking methods cannot be used for endovascular applications. In this environment, using medical imaging scanners to obtain tracking feedback data is mandatory. In addition to being widespread in hospitals, MRI scanners provide a magnetic actuation method without depth limitation $[9,10]$, real-time interventional software architecture [12], X-Ray free unparalleled soft tissue contrast and high precision and high sensitivity tracking [11]. These positive features make the use of MRI systems as a core element for novel interventional platforms a very promising approach.

In vivo computerized navigation of a $1.5 \mathrm{~mm}$ sphere in the carotid artery of a living swine was achieved through proper integration of the different components of a clinical MRI system [13].

This paper presents the integration issues and the medical and technical protocol involved in achieving this proof of concept. It begins with a description of the system architecture followed by the experimental protocol (animal preparation, tracking, trajectory planning). Real-time in vivo navigation is then described followed by sphere release and retrieval techniques.

\section{System Overview}

The interventional procedure is executed using a standard clinical MRI system (Siemens Magnetom Avanto 1.5T, Erlangen, Germany) with real-time feedback capabilities. A ferromagnetic sphere is propelled in the vascular network of a living swine through the application of magnetic gradient pulses using standard imaging magnetic gradient coils already present on all MRI systems [9, 13]. A comprehensive custom software environment for the control, propulsion and tracking is responsible for the device navigation in the swine [11] with a feedback control frequency of 24 $\mathrm{Hz}$. An overview of the real-time pulse sequence used for the device navigation is illustrated on Fig.1. 
For the device to be continuously navigated across a given path, a feedback loop must be present in order to provide a control loop between the decision element, in our case the reconstruction computer, and the execution element, the MRI scanner itself. The real-time feedback capabilities allow the modification of pulse sequence events such as RadioFrequency (RF) pulses, magnetic gradient or ADC on the fly. The developed dedicated software architecture depicted in Fig.1 consists of a path planning module, a tracking module, propulsion and controller module, and a central agent.

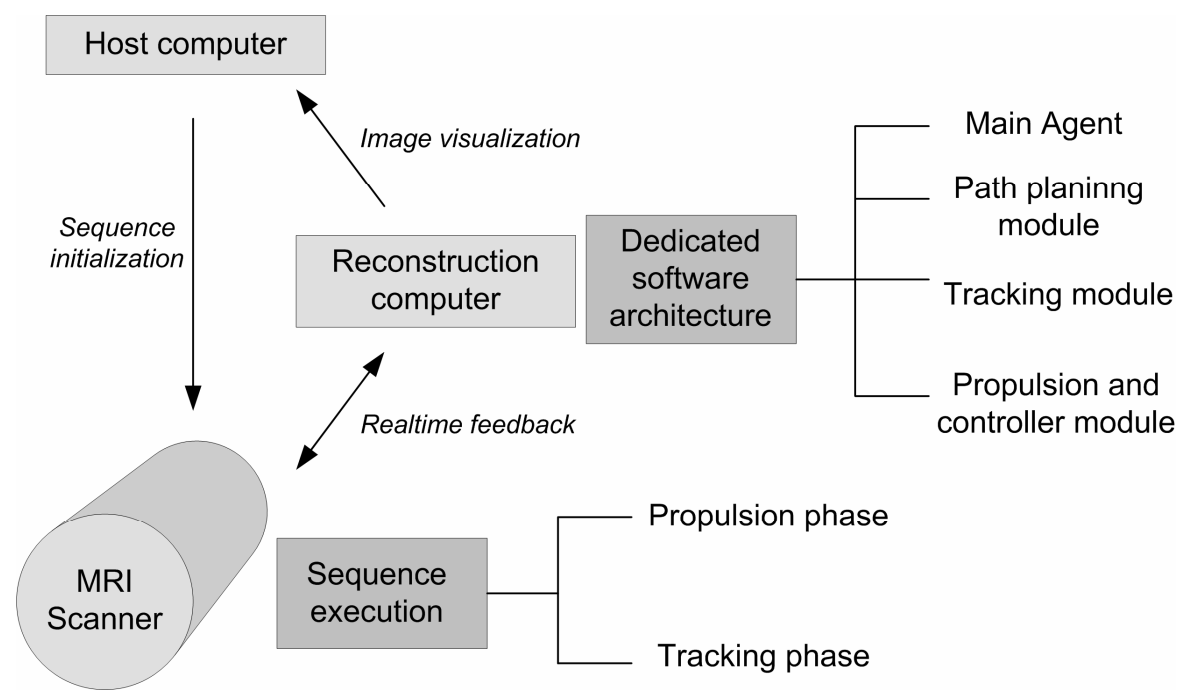

Fig. 1. Overview of the computer architecture incorporated in the standard Siemens environment. The host computer is responsible for the real-time sequence compilation and initialization before being sent for execution on the scanner. This computer also serves as the visualization computer after the image reconstruction step is over. The MRI scanner is the actual hardware that runs the pulse sequence for data acquisition. Finally, the reconstruction computer is responsible for the image generation after the data acquisition step on the scanner is done. In the presented dedicated software environment, a feedback loop exists between the reconstruction computer and the MRI scanner which allows the pulse sequence to be modified on the fly.

All these modules are located in a reconstruction routine in the reconstruction computer. A sequence environment is responsible for the application of the tracking sequence and the propulsion sequence which is executed by the scanner. The reconstruction routine is mainly responsible for the command generation in order to propel the device in a given direction based on the actual position and pre-computed trajectory. Since the device is moved using the magnetic gradient coils already present on the MRI system, the computed command calculated inside the propulsion and controller module is a magnetic gradient amplitude and direction to be applied in the next pulse sequence propulsion phase. The real-time MRI pulse sequence developed is a successive repetition of a propulsion phase which consists of the application of a 
magnetic gradient oriented in space with a given amplitude and an off-resonance tracking phase based on [12]. An overview of the pulse sequence is illustrated in Fig.2.

The image calculation environment consists of two simultaneous running processes. The first process contains the control module which computes the required command to be applied in the next propulsion phase of the real-time sequence. This process is called up through the real-time feedback loop trigger located in the sequence described above. The second process is the tracking module and is called up from the tracking phase of the sequence. It is responsible for the device position computation based on the acquired data of the sequence tracking sequence.

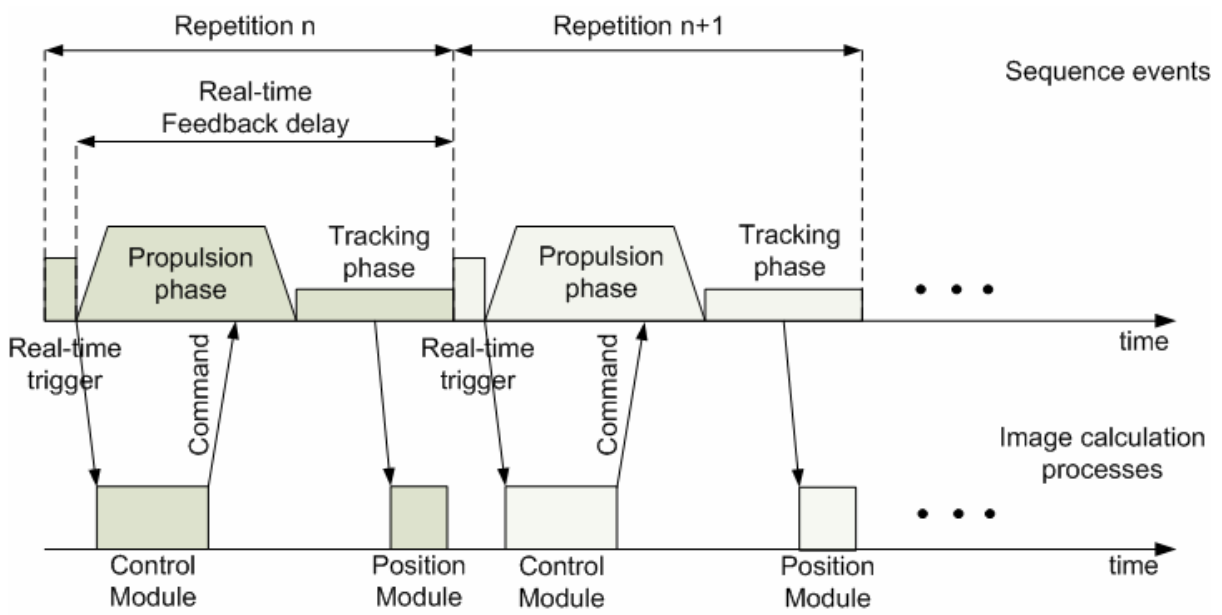

Fig. 2. Overview of the real-time sequence and the image calculation processes for the navigation of the magnetic sphere. A sequence kernel composed of a real-time trigger event, a propulsion phase event and a tracking event is repeated over time. The real-time trigger event starts the control module process for the command generation and the tracking phase calls up the position module process for the device position calculation.

Projections taking advantage of the magnetic field being induced by the sphere are used for tracking [11]. Following the application of a non-selective off-resonance RF pulse, only the spins surrounding the device are excited. Readout gradient are then used to acquire three orthogonal projections that are processed by the tracking module. Once computed, the position is stored for the next command computation in the control module. A central agent is responsible for the overall modules interactions and for the command sending to the running pulse sequence.

The real-time feedback delay depicted in Fig.2 is the minimum time allowed for the command computation and transmission. It is set by the user prior to the intervention. Below this delay, the command computation cannot be completed and the sequence is aborted. Since the sequence tracking phase duration is fixed and lasts $22 \mathrm{~ms}$ for a 3D positioning scheme, the propulsion phase delay is thus only dependent on the real-time feedback delay and is equal to the real-time feedback delay minus the tracking delay. A longer propulsion phase translates into a longer force application time on the device but also in a shorter operating control frequency. Moreover, a 
longer propulsion phase means a longer contribution time of the magnetic gradient coils which could lead to a heating threshold overshoot, preventing the sequence execution.

\section{Interventional Protocol}

\subsection{Animal Preparation}

In vivo experiments are performed on a $25 \mathrm{~kg}$ domestic swine under general anaesthesia (Pentobarbital). This assay study is pre-approved by the animal care and use committee. One 6-F, $80 \mathrm{~cm}$ long introducer catheter (Cook, Bloomington, Indiana) is inserted through a right femoral approach into the proximal portion of the right carotid artery under fluoroscopic guidance (HICOR/ACOM-TOP, Siemens, Erlangen, Germany). A short 5-F introducer is also positioned in the left femoral artery and a $5 \mathrm{~mm} \times 18 \mathrm{~mm}$ angioplasty balloon (AV100, Medtronic, Santa Rosa, CA) is advanced under fluoroscopic guidance in the distal portion of the right common carotid artery $(10 \mathrm{~cm}$ downstream to the tip of the long introducer) over a 0.018" guide wire. The long introducer is used as the release route for the magnetic sphere to be controlled whereas the balloon catheter is used to control the flow and eventually block the sphere in order to facilitate its retrieval upon completion of the control experiments.

The swine is placed in the MRI scanner. A spine with a body array coils are used to collect the MRI signal. The animal is inserted feet first supine and centered inside the MRI bore with respect to its carotid artery. The 6-F inner dilator catheter of the long introducer catheter is used to push a chrome steel sphere inside the long introducer. The sphere is brought in pre-release position $15 \mathrm{~mm}$ before the distal tip of the long introducer using a color marking on the inner dilator as a visual landmark. The balloon catheter is inflated to prevent the sphere from being carried away in case of unplanned problems. The real time MRI sequence is then started and the sphere can be released by pushing the dilator all the way through the long introducer. Once the sphere is released, its movement is dealt with by the control sequence which objective is to navigate it by following the waypoints. After travelling through all of the waypoints, the control sequence is turned off and the tracking data history is saved.

\subsection{Tracking}

Since tracking of the ferromagnetic sphere relies on its magnetic signature, it is critical not to have any other magnetic source present in the imaging volume. An offresonance imaging sequence is used to verify that the volume of interest is free from magnetic perturbation that can interfere with the tracking phase.

The volume is screened to ensure that there is no magnetic perturbation. The device is introduced inside the arteries attached to a catheter and brought as close as possible to the operating region. The tracking sequence is then executed in order to fine tune its parameters like the offset RF frequency, the flip angle and bandwidth per pixel. These parameters depend in fact on the magnetic characteristic and size of the device being used as well as the background tissues where the acquisition is done. 


\subsection{Angiography, Path Planning and Registration}

Once the animal has been tested for magnetic perturbations, a roadmap of the environment and the trajectory for the sphere are determined. First step of the process implies imaging the Region Of Interest (ROI) using a standard MRI angiographic sequence with gadoteridol (ProHance, Bracco Diagnostics, Mississauga, Canada). Threshold filtering is applied on the 3D scan to allow better visualization of the target region before the path-planning step.

In order to determine the path the device will follow, waypoints are placed in the acquired volume (Fig. 3). Eleven waypoints consisting of three coordinates with a back and forth trajectory between the last two coordinates, are set. The next procedure implies transforming these points in the global MRI coordinates axis of reference. The registration software developed as a combination of an MRI sequence and a Matlab script is used to analyze the device position, transform the waypoints coordinates to the MRI frame of reference and display the result over the image acquired earlier. Synchronisation of the waypoint trajectory file with the real actual position of the sphere in reference to the isocenter of the MRI is then performed [14].

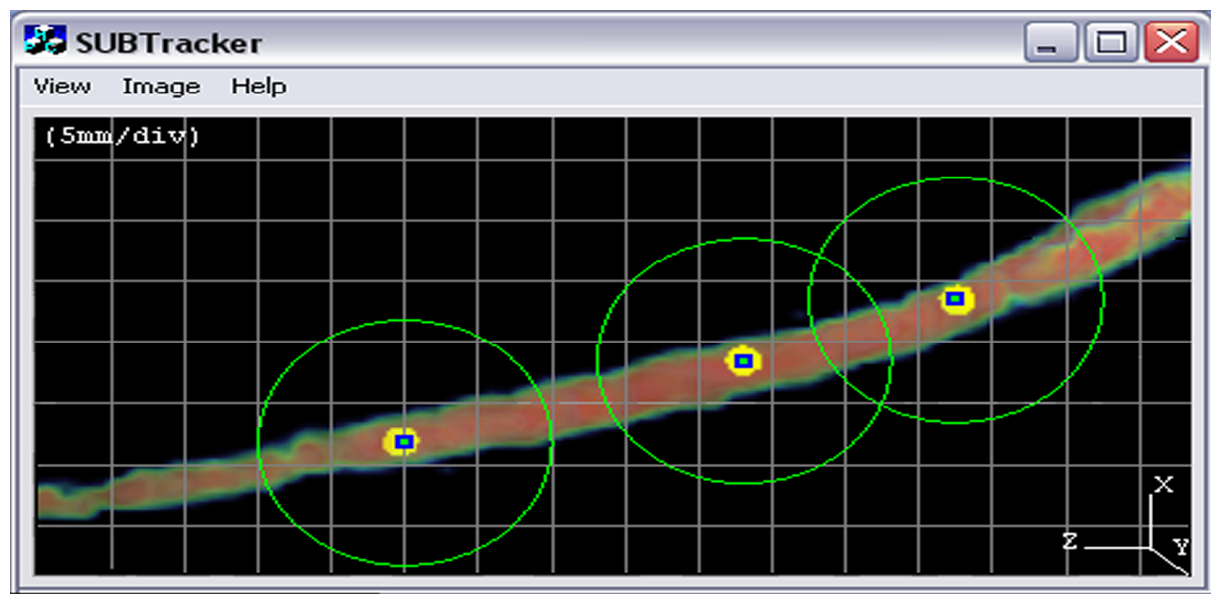

Fig. 3. 3D volume of the carotid artery of the swine filtered at $50 \%$ of the image scalar intensity range. Dots show the waypoints the sphere followed, circles show the precision regions of radius $\varphi$.

\section{Endovascular Navigation}

\subsection{Real-Time Navigation}

Once the waypoints are in the correct axis coordinate system, they are saved in a path file containing all the $3 \mathrm{D}$ positions to be reached during the intervention. Before the sequence is started, the waypoints list is loaded into memory in the control module for fast access during the operation. During the execution, the device's position coordinates computed through the tracking module process are compared with the 
next waypoint to be reached. If the error distance between the bead and the waypoint is comprised in a circle of radius $\varphi$ centered on the waypoint's position (Fig.3.), the waypoint is considered to be reached and the next waypoint is loaded for the destination. The circle radius was set to $\varphi=10 \mathrm{~mm}$ prior to the intervention.

Real-time navigation of the ferromagnetic sphere in the carotid artery of the swine requires a proper real-time feedback delay to be chosen in order to allow both a sufficient propulsion power and a sufficient operating frequency to ensure stability and efficiency for the control loop. Prior to the in vivo intervention, many navigation experiments in MRI phantoms have been realized in order to empirically obtain the optimal real-time feedback delay. A feedback delay of $41 \mathrm{~ms}$ was chosen for the in vivo intervention, leading to a propulsion phase delay of $19 \mathrm{~ms}$ considering a fixed tracking time of $22 \mathrm{~ms}$. The total sequence repetition time is thus $30.6 \mathrm{~ms}$ leading to an achievable operation frequency of $24 \mathrm{~Hz}$ for autonomous navigation. This operation frequency is adequate for device navigation through the selected trajectory described in 3.3. A real-time imaging sequence provided by Siemens (Trufi-irttt) is used to monitor the introduction of the sphere through the long introducer. The realtime control program is then started, deals with the position of the sphere through the 11 waypoints of the trajectory and stabilizes it on the last one. A maximum velocity of $13 \mathrm{~cm} / \mathrm{s}$ was achieved. Retrieval of the sphere is performed using a custom magnetic tipped catheter under Trufi-irttt supervision.

\section{Conclusion}

It was demonstrated that MRI systems provide all the components for implementing a medical device navigation platform (actuation method, imaging of biological tissues, device tracking and computer feedback). The methodology described in the previous sections identifies the main aspects and constraints related with future nanomedical interventional protocols. It addresses the aspects of system architecture design, fast and precise tracking technique, path planning, coordinate system registration as well as device release and recovery.

The long term objective of the project is the spatial control of an agglomeration of magnetic micro/nano devices for targeted delivery of drugs and biosensors. Such agglomeration will be injected as a bolus of a magnetic colloidal suspension that will be carried by the blood flow and guided using external magnetic gradients.

Both the methodology and navigation platform will have to be adapted for that purpose. Due to the scaling laws of magnetic propulsion, special hardware components will have to be developed to generate sufficient magnetophoretic velocity for the micro/nano particles [10]. The tracking scheme will likely change from an Off resonance excitation based technique to a more sensitive $\mathrm{T} 2 *$ contrast method while the injected bolus will get dilute when spreading in the vascular bed. A learning control algorithm will have to be implemented in order to determine an optimal steering gradient combination for targeting. Other improvements will arise from the refinement of the protocol and methods with time and usage. As a matter of fact, in its current state, the protocol that was described in this paper can take several hours to be executed. Many steps during the procedure will have to be made automatic before it 
can gain acceptance from the physicians. Short terms applications of this navigation platform can also be envisioned since small modifications of clinical MRI systems would allow them to navigate readily available medical instruments such as catheters with minimum investment from hospitals.

\section{References}

1. Alksne, J.F., Fingerhunt, A.G.: Magnetically controlled metallic thrombosis of intracranial aneurysms. Bull. Los Angeles Neurol. Soc. 30, 153-155 (1965)

2. Alexiou, C., Arnold, W., Hulin, P., Klein, R.J., Renz, H., Parak, F.G., Bergemann, C., Lubbe, A.S.: Magnetic mitoxantrone nanoparticle detection by histology, X-ray and MRI after magnetic tumor targeting. Journal of Magnetism and Magnetic Materials 225(1-2), 187-193 (2001)

3. Babincova, M., Cicmanec, P., Altanerova, V., Altaner, C., Babinec, O.: AC-magnetic field controlled drug release from magnetoliposomes: Design of a method for site-specific chemotherapy. Bioelectrochemistry 55(1-2), 17-19 (2002)

4. Morales, M.A., Jain, T.K., Labhasetwar, V., Leslie-Pelecky, D.L.: Magnetic studies of iron oxide nanoparticles coated with oleic acid and Pluronic block copolymer. Journal of Applied Physics 97(10) (2005)

5. Viroonchatapan, E., Ueno, M., Sato, H., Adachi, I., Nagae, H., Tazawa, K., Horikoshi, I.: Preparation and characterization of dextran magnetite-incorporated thermosensitive liposomes: an on-line flow system for quantifying magnetic responsiveness. Pharm Res. 12, 1176-1183 (1995)

6. Stereotaxis. Stereotaxis Home. Page last accessed: 20/03/2007, http://www.stereotaxis.com/

7. Yesin, K.B., Exner, P., Vollmers, K., Nelson, B.J.: Design and control of in-vivo magnetic microrobots. In: Duncan, J.S., Gerig, G. (eds.) MICCAI 2005. LNCS, vol. 3749, pp. 819826. Springer, Heidelberg (2005)

8. Yesin, K.B., Vollmers, K., Nelson, B.J.: Modeling and control of untethered biomicrorobots in a fluidic environment using electromagnetic fields. International Journal of Robotics Research 25(5-6), 527-536 (2006)

9. Mathieu, J.-B., Beaudoin, G., Martel, S.: Method of propulsion of a ferromagnetic core in the cardiovascular system through magnetic gradients generated by an MRI system. IEEE Transactions on Biomedical Engineering 53(2), 292-299 (2006)

10. Mathieu, J.-B., Martel, S.: Magnetic Steering of Iron Oxide Microparticles Using Propulsion Gradient Coils in MRI. In: Proceedings of 28th Annual International Conference of the IEEE in Engineering in Medicine and Biology Society EMBS; New York City, USA (2006)

11. Felfoul, O., Mathieu, J.-B., Beaudoin, G., Martel, S.: MR-tracking Based on Magnetic Signature Selective Excitation. IEEE Transactions On Medical Imaging. (Accepted, to be published ref\# TMI-2006-0769) (2007)

12. Chanu, A., Aboussouan, E., Tamaz, S., Martel, S.: Sequence Design and Software Environment for Real-time Navigation of a Wireless Ferromagnetic Device using MRI System and Single Echo 3D Tracking. In: Proceedings of 28th Annual International Conference of the IEEE in Engineering in Medicine and Biology Society EMBS; New York City, USA pp. 1746-1749 (2006) 
13. Martel, S., Mathieu, J.-B., Felfoul, O., Chanu, A., Aboussouan, E., Tamaz, S., Pouponneau, P., Beaudoin, G., Soulez, G., Yahia, L.H., Mankiewicz, M.: Automatic navigation of an untethered device in the artery of a living animal using a conventional clinical magnetic resonance imaging system. Applied Physics Letters 90, 114105 (2007)

14. Aboussouan, E., Martel, S.: High precision absolute positioning of medical instruments in MRI systems. In: Proceedings of 28th Annual International Conference of the IEEE in Engineering in Medicine and Biology Society EMBS, August. 30 - September 3, New York City, USA, pp. 743-746 (2006) 